\title{
Level-of-Service Based Hierarchical Feedback Control Method of Network-Wide Pedestrian Flow
}

\author{
Zhe Zhang, ${ }^{1,2}$ Limin Jia, ${ }^{1,2,3}$ and Yong Qin ${ }^{1,2,3}$ \\ ${ }^{1}$ Traffic and Transportation School, Beijing Jiaotong University, Beijing 100044, China \\ ${ }^{2}$ State Key Laboratory of Rail Traffic Control and Safety, Beijing Jiaotong University, Beijing 100044, China \\ ${ }^{3}$ Beijing Research Center of Urban Traffic Information Sensing and Service Technologies, Beijing Jiaotong University, \\ Beijing 100044, China
}

Correspondence should be addressed to Limin Jia; jialm@vip.sina.com

Received 3 November 2016; Accepted 28 November 2016

Academic Editor: Mohsen Sheikholeslami

Copyright (C) 2016 Zhe Zhang et al. This is an open access article distributed under the Creative Commons Attribution License, which permits unrestricted use, distribution, and reproduction in any medium, provided the original work is properly cited.

\begin{abstract}
Pedestrian flow control is usually used to manage the crowd motion in public facilities to avoid congestion. We propose a networkwide pedestrian flow model based on the modified cell transmission model which describes the link flow as ordinary differential equations. The network flow control model (NFCM) is proposed to limit the number of pedestrians in a network according to the level-of-service requirements; however, the NFCM cannot ensure the uniform link density which is a premise of using NFCM. As a solution, the link flow control model (LFCM) is proposed to adjust the walking speed of pedestrians to realize the uniform link density. The NFCM provides the inputs for the LFCM and the LFCM compensates the deficiency of NFCM. Both NFCM and LFCM control the pedestrian flow in a cooperative way, and thus they form the hierarchical feedback control model (HFCM) of networkwide pedestrian flow. At last, the proposed HFCM is applied to control the crowd of a hall and the comparison of the simulation results in the controlled and uncontrolled scenarios shows that the proposed HFCM has the capability to suggest the optimal link inflows and walking speeds in real time to meet the LOS requirement.
\end{abstract}

\section{Introduction}

1.1. Background. Many public facilities including complex buildings, transportation stations, football stadiums, commercial malls, and other types of facilities have been suffering from very high levels of crowd congestion in peak hours, especially when large public gatherings are held in these facilities [1-3]. A lot of potential risks will increase due to the high level of pedestrian density [4], and the overcrowded situation decreases the overall welfare of pedestrians [5, 6]. Because of the high cost and long period of walking facility reconstruction, facility managers prefer to use some organization strategies to control pedestrian flow $[7,8]$, such as limiting the inflow of facilities [9] and limiting the walking space by using handrails [10].

From the viewpoint of pedestrians, poor execution of crowd flow control strategy in facilities can put the pedestrians in crowded situation which may reduce the comfort level or cause public accidents including crowd crush, injuries, and fatalities. From the viewpoint of managers, overcrowded pedestrian flow can lead to excessive use of facility capacity, which creates a poor public environment which may destroy the customer experience and contributes towards a lower level of service in turn. The optimal pedestrian flow control strategy is to transport most pedestrians while the security and level of service (LOS) are accepted [9]. Furthermore, the pedestrian flow control strategy is produced based on the managers' experience for now. The absence of a pedestrian flow control system tends to result in overcrowded facilities because of the stochastic nature of traffic demand and behavior. Therefore, intelligent control of pedestrian flow in public facilities is exigently needed.

Further, a video surveillance system has been built [11] in public facilities to enhance the level of safety management; at the same time, it realizes the real-time acquisition of pedestrian density and walking speed [12]. However, the pedestrian flow data captured by videos or sensors is still not used scientifically for pedestrian flow control, and thus the 
main objective of this paper is to introduce a pedestrian flow control model to provide crucial support towards meeting the LOS requirement of pedestrians in everyday operation. A literature review is provided and the contributions of the paper are discussed in the next section.

1.2. Related Literature. Many methods supporting pedestrian flow control and crowd management have been proposed. The majority of these works can be broadly categorized as performance-based method, network-based model, and control theory-based model.

The performance-based method is to evaluate the traffic performance of pedestrian network after simulating the pedestrian flow under self-defined scenarios. With regard to the method, a hybrid Petri nets-based model has been proposed to evaluate the effect of the network layout or design of railway transit station on the crowd safety [13], and a system dynamics based tool has been developed to evaluate the effect of facility allocation plans on the waiting time of passengers [14]. A state-dependent queuing model has been proposed to evaluate the width of corridor on the designed LOS [15], which is based on the standard from [16]. Microscopic crowd simulation models such as agent-based model [17], cellular automata model [10], social force model [18], and lattice gas model [19] have been used to evaluate the performance of walking facilities under different scenarios, and these works can give some suggestions about how to design the facility layout or how to influence the pedestrians' behavior to improve the LOS or service capacity. Models listed in the above works are mainly developed to analyze the effect of network layout and design on the efficiency of pedestrian movement and they cannot provide feasible strategies for managing the crowd directly before simulating the pedestrian flow. These methods have obvious limitations, especially when a large number of crowd control or network optimization plans are required. Furthermore, the success of the above methods is dependent on a limited number of crowd control plans. A large number of crowd management plans may result in a complicated and time-consuming evaluation process, which may lead to the impossible realization of the real-time pedestrian flow control.

The network-based models usually determine optimal routes to which pedestrians should be guided within an existing physical environment and the optimal service and capacity allocation plan of facilities. With regard to the network-based model, an M/G/C/C state-dependent queuing network-based model was also proposed to find the optimal width of corridors to minimize the blocking probability of pedestrian network [20]. A network-based facility allocation model for optimizing passenger flows within a hub has been proposed to reduce the transit delays and improve the LOS of hub facilities [21]. In order to support efficient crowd movement in a network, a network optimization-based approach for crowd management has been proposed to compute the optimal width of corridors and the feasible network configuration to realize the optimal network performance under the budget constraints [22]. However, it is infeasible to increase facility capacities or build new service facilities due to the high cost and long period of reconstruction. Passenger flow control strategies can relieve some demand pressure on the downstream facility by adjusting the passenger inflow of subway stations, and thus a queue-network-based passenger flow control model was proposed by [9] to solve the severe congestion problem in subway stations, and a pedestrian flow optimization method based on $\mathrm{M} / \mathrm{G} / \mathrm{C} / \mathrm{C}$ queue network was also developed to maximize the pedestrian flow rate in evacuation network [23]; however, the queue-network-based control model can only control the inflow of network and has little ability to suggest the real-time optimal inflow of various facilities and the optimal velocities before modification.

The performance-based models and network-based models examine the pedestrians flow in networks from a simulation and analysis point of view, which may be not suitable for real-time and intelligent implementation. The control theory-based models including sliding mode control and feedback control have been proposed to control pedestrian flow based on the real-time state information. The feedback control models have been proposed to support the smooth evacuation in rooms [24, 25], corridors [26], and networks [27] based on the linear or nonlinear ordinary differential equation model (ODE) and hyperbolic partial differential equations (PDEs). Sliding mode control is also used to control the crowd evacuation [28] and avoid the congestion in bottleneck of corridors [29] based on PDEs.

To the best of our knowledge, there is little research to bridge the pedestrian flow control and the LOS requirement. Few studies have performed a real-time control of networkwide pedestrian flow to satisfy the LOS requirement. The realtime crowd control methods described in the previous works are designed mainly for use in crowd evacuation. However, they do not provide strategies for controlling the networkwide pedestrian flow in everyday operation. Accurate determination of the optimal pedestrian flow control to meet LOS requirement has been rare. This study provides a basis for addressing these issues.

1.3. Contributions. We define pedestrian flow control as the rational administration of the pedestrian movement and traffic demand to generate adequate performance of pedestrian network to balance the benefits of pedestrians and managers. The present research demonstrates a cell transmission based network-wide pedestrian flow model and a hierarchical feedback control model to find the optimal pedestrian flow control plan based on the LOS requirement. The proposed research contributes to the state-of-the-art related pedestrian flow control research in three ways:

(1) The links in the pedestrian network are taken as the minimal analytical units, and a network-wide pedestrian flow model has been proposed in a macroscopic sense, wherein ordinary differential equations (ODEs) for each link are obtained based on the modified cell transmission model. The collection of these ODEs is used scientifically to define the state-space model of the network-wide pedestrian flow, and this modeling approach leads to the easier development of pedestrian flow control algorithm. 
(2) A hierarchical feedback control model (HFCM) of pedestrian flow is developed to improve the LOS of pedestrian network, which is a rare try to link the pedestrian flow control with LOS and service capacity requirements. The service capacity of pedestrian network is maximized under the limits of LOS in the control process. The above control objective balances the benefits of managers and pedestrians. Therefore, the proposed control model can meet the demand of pedestrians and managers at the same time.

(3) The network flow control model (NFCM) can suggest the optimal link inflows to satisfy the LOS but cannot ensure the uniform link density, which is the premise of using this model. Therefore, a link flow control model (LFCM) is proposed firstly to realize the uniform density in links such that the NFCM controls the network-wide flow state more precisely. Therefore, the NFCM and LFCM control the network-wide pedestrian flow in a cooperative way and constitute our HFCM.

The rest of the paper is organized as follows. Section 2 builds the network-wide pedestrian flow model by which the pedestrian flow in the network is simulated. Section 3 describes the methodology used to control the network-wide pedestrian flow based on the LOS requirements. Section 4 describes the results of an experiment and a case study that were carried out to verify the effectiveness of the proposed control method. Finally, conclusions and directions for future studies are made.

\section{Network-Wide Pedestrian Flow Model}

The microscopic models are best suited for generating more accurate and detailed description of pedestrians flow modeling and route choice characteristic, but unsuitable for network flow simulation due to more complex structure data input, more computing time, and developing efforts required. Therefore, in this paper, the pedestrian flow is simulated by a macroscopic model which is similar to the vehicular traffic flow model [30] because the macroscopic models can give us a big-picture averaged view of the network-wide pedestrian flow state. In this section, an analytical model for simulating network-wide pedestrian flow is proposed and described as the collection of ordinary differential equations (ODEs). The inputs of the ODEs are generated by the cell transmission model (CTM) proposed by Daganzo [31] and the modified CTM (MCTM) proposed by Muñoz et al. [32].

Let $G=(N, A)$ be a directed pedestrian network defined by a set $N$ of nodes and a set $A$ of directed links. The links denote the walking facilities with holding capacity such as corridors and stairways and the nodes denote the source node and sink node of each link. We call the current pedestrian density and maximum pedestrian density of link the link density and maximum link density, respectively. Similarly, we call the current walking speed and maximum walking speed of link the link speed and maximum link speed, respectively. Let $\rho_{a}$ and $\rho_{a}^{m}$ be the current and maximum link density of link $a$, respectively. The link density can be observed or calculated by probes such as sensors and videos. It can be obtained that $0 \leq \rho_{a} \leq \rho_{a}^{m}$. Let $v_{a}$ and $v_{a}^{m}$ be the link speed and maximum link speed of link $a$, respectively. It can be obtained that $0 \leq v_{a} \leq v_{a}^{m}$. According to the relationship between speed and density, the link speed $v_{a}$ can be described as follows:

$$
v_{a}=v_{a}^{m} f\left(\frac{\rho_{a}}{\rho_{a}^{m}}\right),
$$

where $f(\cdot)$ is a decreasing function and $f(0)=1$ and $f(1)=$ 0 . Equation (1) ensures that the pedestrians can walk at the free speed when there are a small number of pedestrians in link $a$ and they stop walking when the number of pedestrians in link $a$ has reached the holding capacity. Let $L_{a}$ and $W_{a}$ be the length and width of link $a$; the hydrodynamic pedestrian flow in link $a q_{a}$ can be described as the following equation:

$$
q_{a}=W_{a} \rho_{a} v_{a}^{m} f\left(\frac{\rho_{a}}{\rho_{a}^{m}}\right) .
$$

Let $q_{a}^{\text {in }}$ and $q_{a}^{\text {out }}$ be the current inflow and outflow of link $a$, respectively. Using the mass conservation law, the derivation of link density with respect to time $\dot{\rho}_{a}$ can be described as the following ODE:

$$
\dot{\rho}_{a}=\frac{q_{a}^{\text {in }}-q_{a}^{\text {out }}}{L_{a} W_{a}}
$$

In order to simulate the pedestrian flow in uncontrolled scenario, the value of $q_{a}^{\text {in }}$ and $q_{a}^{\text {out }}$ should be determined. In uncontrolled scenario, because of the urgent willingness of arriving at destinations, pedestrians want to enter and leave the links at the maximum inflow $q_{a}^{m \text { in }}$ and outflow $q_{a}^{\text {mout }}$. The cell transmission model [31] (CTM) is used in this paper to determine the value of $q_{a}^{\text {min }}$ and $q_{a}^{\text {mout }}$. The CTM is proposed by Daganzo for approximating the Lighthill and Whitham (1955) and Richards (1956) [30] hydrodynamic traffic flow model (LWR model):

$$
\frac{\partial \rho(x, t)}{\partial t}+\frac{\partial q(x, t)}{\partial x}=0,
$$

where $\rho(x, t)$ denotes the density at place $x$ and time $t$ and $q(x, t)$ is the traffic flow. The CTM divides the links into many cells with the same length and simulates the traffic flow by defining the flow between cells [31]. Compared to the CTM, the modified cell transmission model (MCTM) proposed by Muñoz et al. [32], which discretizes the LWR model (or its simplified version) in both time and space, was shown to be computationally efficient and easy to analyze many important traffic phenomena, such as queue buildup and dissipation [33]. In MCTM, the nonuniform cell lengths are acceptable. Also, the pedestrian CTM has been developed to simulate the pedestrian flow in public facilities and the accuracy results about LOS [34] can be obtained [35]; the CTM was also used to control the vehicular-pedestrian mixed flows in the evacuation zone [36]. All above works indicate that the CTM can simulate the traffic flow in roadway network as well as the pedestrian flow in the network of corridors. By taking each 


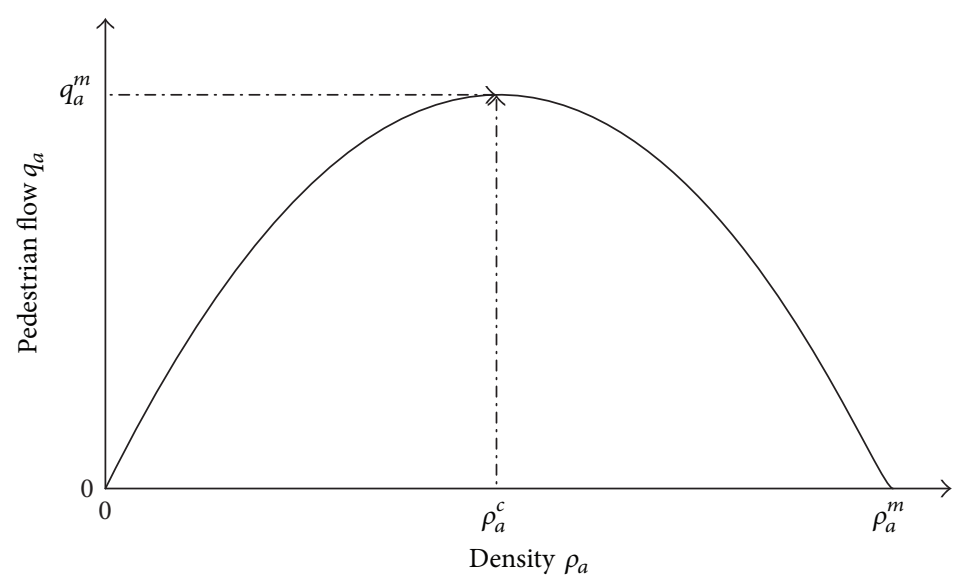

Figure 1: Pedestrian fundamental diagram: an example.

link in the pedestrian network as a cell in MCTM, we can use the MCTM and pedestrian flow fundamental diagram to simulate the network-wide pedestrian flow. Within a link of pedestrian network, pedestrians are assumed to be homogeneously distributed, and their movements are not modeled explicitly.

The hydrodynamic outflow capacity (HOC) can be defined as the maximum number of pedestrians leaving links per unit time and the hydrodynamic inflow capacity (HIC) can be defined as the maximum number of pedestrians entering links per unit time according to the fundamental diagram [34]. In the pedestrian flow fundamental diagram, as shown in Figure 1, there is a critical density $\rho_{a}^{c}$ to maximize the pedestrian flow in link $a q_{a}$ and it divides the link density domain into two regimes. For the link density $\rho_{a} \leq \rho_{a}^{c}$, the increase in link density leads to increased hydrodynamic flow and is thus denoted as the free-flow regime. On the contrary, the congested regime comprises the values of link density $\rho_{a}$ greater than $\rho_{a}^{c}$, as an increase in link density in this domain leads to a decrease in hydrodynamic flow. According to the fundamental diagram, if the link density $\rho_{a} \leq \rho_{a}^{c}$, the HOC of link $a q_{a}^{\text {hoc }}$ is equal to the hydrodynamic flow in link $a$; if the link density $\rho_{a}>\rho_{a}^{c}$, the HOC of link $a q_{a}^{\text {hoc }}$ is equal to the maximum hydrodynamic flow in link $a q_{a}^{m}$; that is,

$$
q_{a}^{\text {hoc }}= \begin{cases}q_{a}, & \text { if } \rho_{a} \leq \rho_{a}^{c}, \\ q_{a}^{m}, & \text { otherwise. }\end{cases}
$$

Similarly, if the density of the downstream link $b \rho_{b} \leq \rho_{b}^{c}$, the HIC of link $b q_{b}^{\text {hic }}$ is equal to the maximum hydrodynamic flow in link $b q_{b}^{m}$; if the link density $\rho_{b}>\rho_{b}^{c}$, the HIC of link $b q_{b}^{\text {hic }}$ is equal to the hydrodynamic flow in link $b q_{b}$; that is,

$$
q_{b}^{\text {hic }}= \begin{cases}q_{b}^{m}, & \text { if } \rho_{b} \leq \rho_{b}^{c} \\ q_{b}, & \text { otherwise. }\end{cases}
$$

As in the MCTM proposed by Muñoz et al. [32], a sending and receiving capacity is defined for each link, representing the maximum number of pedestrians who can leave and enter a link per unit time, respectively. The sending capacity of link $a$ is determined by the minimum value between the number of pedestrians in link $a N_{a}$ and the HOC of link $a q_{a}^{\text {hoc }}$; that is,

$$
S_{a}=\min \left\{N_{a}, q_{a}^{\text {hoc }}\right\} .
$$

The receiving capacity of downstream link $b R_{b}$ is determined by the minimum value between the available holding capacity of link $b M_{b}$ and the HIC of link $b q_{b}^{\text {hic }}$; the available holding capacity of link $b C_{b}$ is the difference between the holding capacity of link $b C_{b}$ and the number of pedestrians in link $b N_{b}$ at time $t$; that is, $M_{b}=C_{b}-N_{b}$. Therefore,

$$
R_{b}=\min \left\{M_{b}, q_{b}^{\text {hic }}\right\} .
$$

The maximum outflow of link $a$ is determined by the sending capacity of link $a$ and the receiving capacity of downstream link $b R_{b}$; that is,

$$
q_{a}^{\text {mout }}=\min \left\{S_{a}, R_{b}\right\} .
$$

The above equation describes the pedestrian flow between two interconnected links in series topology, as indicated by Figure 2(a). In the pedestrian network, there are also merge and split topologies [37], as indicated by Figures 2(b) and 2(c). Tampère et al. [38] established the split model and merge model of traffic flow based on Daganzo's merge and split models [31] and the merge distribution schemes of Jin and Zhang [39] and of Ni and Leonard II [40]. The link outflow can be got by maximizing the total flow in the merge and split node.

For the merge topology as indicated by Figure 2(b), the merge flow can be determined by solving the following linear programming problem:

$$
\begin{array}{ll}
\max & q^{\text {mout }}=q_{a}^{\text {mout }}+q_{b}^{\text {mout }} \\
\text { s.t. } & q_{a}^{\text {mout }} \leq S_{a} \\
& q_{b}^{\text {mout }} \leq S_{b} \\
& q_{a}^{\text {mout }}+q_{b}^{\text {mout }} \leq R_{c} .
\end{array}
$$




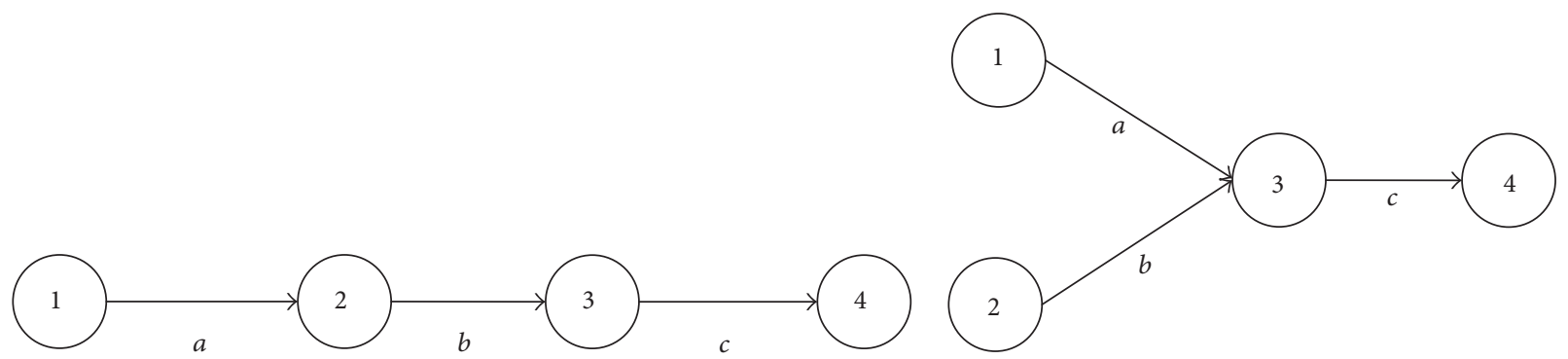

(a) Series topology

(b) Merge topology

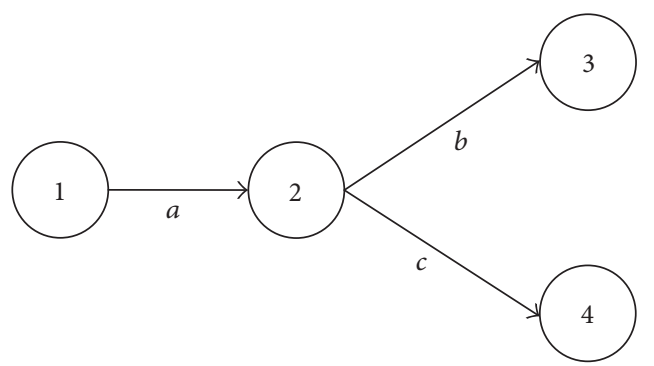

(c) Split topology

FIGURE 2: Series, merge, and split topology in pedestrian network.

As can be seen from (11), the objective function $q^{\text {mout }}=$ $\min \left\{S_{a}+S_{b}, R_{c}\right\}$. All pedestrians in link $a$ and link $b$ can enter the link $c$ if the sending capacity of incoming links $a$ and $b$ does not exceed the receiving capacity of link $c R_{c}$; otherwise, the receiving capacity of link $c R_{c}$ is allocated proportionally to the two incoming links. A level of reduction $\alpha$ has been introduced to represent the proportion of the sending capacity of links $a$ and $b$ being transferred to link $c$ when the receiving capacity of link $c R_{c}$ cannot handle the incoming pedestrians flow $S_{a}+S_{b}$ :

$$
\alpha=\frac{R_{c}}{S_{a}+S_{b}} .
$$

The pedestrian flow from link $a$ to link $c q_{a}^{\text {mout }}$ and the pedestrian flow from link $b$ to link $c q_{b}^{\text {mout }}$ can be described as follows:

$$
\begin{aligned}
& q_{a}^{\text {mout }}=\min \{1, \alpha\} S_{a}, \\
& q_{b}^{\text {mout }}=\min \{1, \alpha\} S_{b} .
\end{aligned}
$$

For the split topology as indicated by Figure 2(c), let $P_{b}$ and $P_{c}$ be the transfer probability from link $a$ to link $b$ and link $c$, respectively, and the flow constraints can be described as follows:

$$
\begin{aligned}
& q_{a}^{\text {mout }}=q_{a b}^{\text {mout }}+q_{a c}^{\text {mout }} \leq S_{a}, \\
& q_{a b}^{\text {mout }}=P_{b} q_{a}^{\text {mout }} \leq R_{b}, \\
& q_{a c}^{\text {mout }}=P_{c} q_{a}^{\text {mout }} \leq R_{c} .
\end{aligned}
$$

Based on (10) and (15), the maximum outflow of link $a$ can be described as follows:

$$
q_{a}^{\text {mout }}=\min \left\{S_{a}, \frac{R_{b}}{P_{b}}, \frac{R_{c}}{P_{c}}\right\} .
$$

Then, the pedestrian flow from link $a$ to link $b$ and links $c q_{a b}^{\text {mout }}$ and $q_{a c}^{\text {mout }}$ can be described as follows:

$$
\begin{aligned}
& q_{a b}^{\text {mout }}=\min \left\{1, \alpha_{1}, \alpha_{2}\right\} P_{b} S_{a}, \\
& q_{a c}^{\text {mout }}=\min \left\{1, \alpha_{1}, \alpha_{2}\right\} P_{c} S_{a},
\end{aligned}
$$

where $\alpha_{1}$ and $\alpha_{2}$ are defined as follows:

$$
\begin{aligned}
& \alpha_{1}=\frac{R_{b}}{P_{b} S_{a}} \\
& \alpha_{2}=\frac{R_{c}}{P_{c} S_{a}} .
\end{aligned}
$$

According to the inflow and outflow characteristics of links, the links in the pedestrian network $G=(N, A)$ are divided into four groups: the link group without inflow and with outflow at time $t A_{o}(t)$, the link group with inflow and outflow at time $t A_{d}(t)$, the link group with inflow and without outflow at time $t A_{i}(t)$, and the link group without inflow and outflow at time $t A_{e}(t)$. The link $a$ can belong to different link group at different time $t$. The four link groups in the pedestrian network $G=(N, A)$ can be described as follows:

$$
\begin{aligned}
& A_{o}(t)=\left\{a \mid q_{a}^{\text {min }}=0, q_{a}^{\text {mout }}>0\right\}, \\
& A_{d}(t)=\left\{a \mid q_{a}^{\text {min }}>0, q_{a}^{\text {mout }}>0\right\}, \\
& A_{i}(t)=\left\{a \mid q_{a}^{\text {min }}>0, q_{a}^{\text {mout }}=0\right\}, \\
& A_{e}(t)=\left\{a \mid q_{a}^{\text {min }}=0, q_{a}^{\text {mout }}=0\right\} .
\end{aligned}
$$

The link $a$ may belong to different link groups at different time $t$ because the link inflow and outflow are time-dependent variable. 


\section{Pedestrian Flow Control Model}

When the capacity of pedestrian network cannot satisfy the traffic demand, crowd congestion will occur. In order to deal with the congestion and improve the LOS of pedestrian network, the control measures such as limiting the pedestrian inflow or the variable speed limiting will be conducted with design and usage of devices, such as speakers and video displays, to guide the pedestrians [41]. In this section, a hierarchical feedback control model (HFCM) of network-wide pedestrian flow is proposed to satisfy the LOS requirements. The proposed HFCM comprises two models: network flow control model (NFCM) and link flow control model (LFCM). The NFCM produces the inflow and outflow control plan of each link to satisfy the LOS requirement and the LFCM produces the walking speed control plan which realizes uniform link density after receiving the control inputs from the NFCM.

3.1. Network Flow Control Model. In peak hours, there is a conflict between the management objective of network managers and the satisfaction of pedestrians. First, network managers will control the pedestrian inflow of each link if the LOS of links becomes lower. However, the pedestrian flow control measures may increase the delay time of pedestrians and reduce their satisfaction. In order to measure the LOS of links, the link densities are usually considered [42]. On the other hand, pedestrians want to spend less time in walking to destination $[43,44]$.

If the targets of network managers and pedestrians are achieved at the same time, the density of the objective LOS, which can maximize the link flow, can be considered as the objective density value of pedestrian flow control because the objective density balances the profits of managers and passengers. Let $\left[\rho_{a}^{l}, \rho_{a}^{u}\right]$ be the density domain for the required LOS of link $a$; according to the fundamental diagram of pedestrian flow as indicated by Figure 1, the objective density of link $a \rho_{a}^{t}$ can be described as follows:

$$
\rho_{a}^{t}= \begin{cases}\rho_{a}^{u}, & \text { if } \rho_{a}^{u} \leq \rho_{a}^{c}, \\ \rho_{a}^{c}, & \text { if } \rho_{a}^{l} \leq \rho_{a}^{c} \leq \rho_{a}^{u}, \\ \rho_{a}^{l}, & \text { if } \rho_{a}^{l} \geq \rho_{a}^{c} .\end{cases}
$$

If the LOS of links is lower than the required LOS, the control measures should be taken to achieve the convergence of the link density $\rho_{a}$ to the objective density $\rho_{a}^{t}$ for the link $a \in$ $A_{d}(t) \cup A_{i}(t)$, to achieve the convergence of the link density $\rho_{a}$ to the 0 for the link $a \in A_{o}(t)$, and to maximize the number of pedestrians served by the network per unit time $Q_{s}$ while realizing the two above objectives. The link $a \in A_{e}(t)$ has no inflow and outflow and thus can be deleted from the network at time $t$, which reduces the computing time of the proposed control algorithm. Let the binary variable $\sigma_{a}$ denote the link with or without inflow, $\sigma_{a}=1$ denote the link $a \in A_{d}(t) \cup A_{i}(t)$, and $\sigma_{a}=0$ denote the link $a \in A_{o}(t)$. The exponential convergence of link densities to the objective densities can be realized if the derivation of link density with respect to time $\dot{\rho}_{a}$ satisfies the following equation:

$$
\dot{\rho_{a}}=-k_{a}\left(\rho_{a}-\sigma \rho_{a}^{t}\right)
$$

where $k_{a}$ is the control gain and is greater than zero. Substituting (3) into (20), the following equation can be obtained:

$$
\frac{q_{a}^{\text {in }}-q_{a}^{\text {out }}}{L_{a} W_{a}}=-k_{a}\left(\rho_{a}-\sigma \rho_{a}^{t}\right) .
$$

The controlled inputs of the network flow control model are the inflow and outflow of links $q_{a}^{\text {in }}$ and $q_{a}^{\text {out }}$. Therefore, (21) represents the feedback linearization of the system of statespace equation (3) [27] and denotes the equation constraints on the control inputs $q_{a}^{\text {in }}$ and $q_{a}^{\text {out }}$.

As described in Section 2, the maximum value of the inflow and outflow of links occurs in uncontrolled pedestrian flow; therefore, the inflow and outflow of links $q_{a}^{\text {in }}$ and $q_{a}^{\text {out }}$ are greater than or equal to zero and lower than or equal to the maximum inflow and outflow in uncontrolled pedestrian flows $q_{a}^{\text {min }}$ and $q_{a}^{\text {mout }}$. All in all, the bound constraint of the control inputs can be described as follows:

$$
\begin{aligned}
& 0 \leq q_{a}^{\text {in }} \leq q_{a}^{\text {min }} \\
& 0 \leq q_{a}^{\text {out }} \leq q_{a}^{\text {mout }} .
\end{aligned}
$$

From the analysis of control objective, the number of pedestrians served by the network per unit time $Q_{s}$ (service capacity) should be maximized in the control process. The service capacity $Q_{s}$ is equal to maximizing the number of pedestrians leaving the network per unit time. Let $Q_{e}$ be the number of pedestrians entering the network per unit time; the following equation can be got by adding (21) of each link in the pedestrian network:

$$
Q_{e}-Q_{s}=\sum_{a \in A}-k_{a}\left(\rho_{a}-\sigma \rho_{a}^{t}\right)
$$

Then, $Q_{e}=Q_{s}+\sum_{a \in A}-k_{a}\left(\rho_{a}-\sigma \rho_{a}^{t}\right)$; because the second part of the right hand of this equation is a variable independent of control inputs, maximizing service capacity $Q_{s}$ is equal to maximizing the number of pedestrians entering the network per unit time $Q_{e}$; that is, $\max Q_{s} \cong \max Q_{e}$.

At every time instant, the control inputs satisfying the equation constraint (21) and bound constraint (22) should be solved to maximize the number of pedestrians entering the network per unit time $Q_{e}$. The equation constraint (21) is linear and the number of pedestrians entering the network per unit time $Q_{e}$ is also the linear sum of the inflows of 
source links in pedestrian network. Therefore, the networkwide pedestrian flow control model can be described as the following linear programming problem (LP):

$$
\begin{array}{ll}
\max & Q_{e} \\
\text { s.t. } & \frac{q_{a}^{\text {in }}-q_{a}^{\text {out }}}{L_{a} W_{a}}=-k_{a}\left(\rho_{a}-\sigma \rho_{a}^{t}\right) \\
& 0 \leq q_{a}^{\text {in }} \leq q_{a}^{\text {min }} \\
& 0 \leq q_{a}^{\text {out }} \leq q_{a}^{\text {mout }} .
\end{array}
$$

The large control gain $k_{a}$ may make the above LP problem become infeasible. In order to select the proper control gains, we can define a high value $k_{h}$ as the initial control gain of the LP problem, and then we can get the infeasible solution $q_{s}=\left(q_{a}^{\text {in }}, q_{a}^{\text {out }}, \ldots\right)$ which is greater than the upper bounds of the control input $q^{u}=\left[q_{a}^{\text {min }}, q_{a}^{\text {mout }}, \ldots\right]$. Let the scaling factor $\mu=\max \left(q_{s} / q^{u}\right)$, and then the proper control gain $k_{a}=k_{h} / \mu$, which will make the LP problem become feasible.

3.2. Link Flow Control Model. The pedestrians are assumed to be homogeneously distributed all the time in NFCM. However, the NFCM may lead to the nonuniform link density because the minimal control unit of NFCM is the links of pedestrian network. Therefore, the link flow control model is proposed in this section to realize the uniform link density with the control inputs from the $\operatorname{NFCM} q_{s}=\left(q_{a}^{\text {in }}, q_{a}^{\text {out }}, \ldots\right)$. The solution to the LP problem has given the details about the inflow and outflow of link $a q_{a}^{\text {in }}, q_{a}^{\text {out }}$. We divide the unidirectional links into $n$ sections with the same length $l_{a}$. Let $q_{a}^{i}$ and $\rho_{a}^{i}$ be the inflow and density of link section $i$, respectively. Similarly, let $\dot{\rho}_{a}^{i}$ be the derivation of section density with respect to time and $\rho_{a}^{i}$ can be formulated as

$$
\begin{aligned}
\dot{\rho}_{a}^{1} & =\frac{q_{a}^{\text {in }}-q_{a}^{2}}{l_{a} W_{a}}, \\
\dot{\rho}_{a}^{i} & =\frac{q_{a}^{i}-q_{a}^{i+1}}{l_{a} W_{a}}, \text { for } 1<i<n, \\
\dot{\rho}_{a}^{n} & =\frac{q_{a}^{n}-q_{a}^{\text {out }}}{l_{a} W_{a}} .
\end{aligned}
$$

The inflow of section $i q_{a}^{i}$ can be described as $q_{a}^{i}=$ $\rho_{a}^{i-1} v_{a}^{i} f\left(\rho_{a}^{i-1} / \rho_{a}^{m}\right)$ if the section density is assumed to be uniform [26]. $v_{a}^{i}$ is the controlled walking speed of pedestrians in section $i$ and $0 \leq v_{a}^{i} \leq v_{a}^{f}$. Equation (26) can be formulated as follows:

$$
\begin{aligned}
\dot{\rho_{a}^{1}} & =\frac{q_{a}^{\text {in }}-W_{a} \rho_{a}^{1} v_{a}^{1} f\left(\rho_{a}^{1} / \rho_{a}^{m}\right)}{l_{a} W_{a}}, \\
\dot{\rho}_{a}^{i} & =\frac{W_{a} \rho_{a}^{i-1} v_{a}^{i-1} f\left(\rho_{a}^{i-1} / \rho_{a}^{m}\right)-W_{a} \rho_{a}^{i} v_{a}^{i} f\left(\rho_{a}^{i} / \rho_{a}^{m}\right)}{l_{a} W_{a}},
\end{aligned}
$$$$
\text { for } 1<i<n \text {, }
$$

$$
\dot{\rho}_{a}^{n}=\frac{W_{a} \rho_{a}^{n-1} v_{a}^{n-1} f\left(\rho_{a}^{n-1} / \rho_{a}^{m}\right)-q_{a}^{\text {out }}}{l_{a} W_{a}} .
$$

If the link density is nonuniform, the link flow control measures should be applied to realize the density difference between link sections $D^{i}$ to zero; for link $a$, the density difference $D_{a}^{i}$ can be described as follows:

$$
D_{a}^{i}=\rho_{a}^{i}-\rho_{a}^{i+1}=0
$$

The exponential convergence of density difference between sections to zero can be realized if the derivation of section density difference with respect to time $D_{a}^{i}$ satisfies the following equation:

$$
\dot{D}_{a}^{i}=-k_{a}^{i} D_{a}^{i}
$$

where $k_{a}^{i}$ is the control gains of the link flow control model. Substituting (27) into (29), we can obtain the following equations:

$$
\begin{gathered}
\frac{\left(q_{a}^{\text {in }}-W_{a} \rho_{a}^{1} v_{a}^{1} f\left(\rho_{a}^{1} / \rho_{a}^{m}\right)\right)-\left(W_{a} \rho_{a}^{1} v_{a}^{1} f\left(\rho_{a}^{1} / \rho_{a}^{m}\right)-W_{a} \rho_{a}^{2} v_{a}^{2} f\left(\rho_{a}^{2} / \rho_{a}^{m}\right)\right)}{l_{a} W_{a}}=-k_{a}^{1}\left(\rho_{a}^{1}-\rho_{a}^{2}\right), \\
\frac{\left(W_{a} \rho_{a}^{i-1} v_{a}^{i-1} f\left(\rho_{a}^{i-1} / \rho_{a}^{m}\right)-W_{a} \rho_{a}^{i} v_{a}^{i} f\left(\rho_{a}^{i} / \rho_{a}^{m}\right)\right)-\left(W_{a} \rho_{a}^{i} v_{a}^{i} f\left(\rho_{a}^{i} / \rho_{a}^{m}\right)-W_{a} \rho_{a}^{i+1} v_{a}^{i+1} f\left(\rho_{a}^{i+1} / \rho_{a}^{m}\right)\right)}{l_{a} W_{a}}=-k_{a}^{i}\left(\rho_{a}^{i}-\rho_{a}^{i+1}\right), \\
\quad 1<i<n-1, \\
\frac{\left(W_{a} \rho_{a}^{n-2} v_{a}^{n-2} f\left(\rho_{a}^{n-2} / \rho_{a}^{m}\right)-W_{a} \rho_{a}^{n-1} v_{a}^{n-1} f\left(\rho_{a}^{n-1} / \rho_{a}^{m}\right)\right)-\left(W_{a} \rho_{a}^{n-1} v_{a}^{n-1} f\left(\rho_{a}^{n-1} / \rho_{a}^{m}\right)-q_{a}^{\text {out }}\right)}{l_{a} W_{a}}=-k_{a}^{n-1}\left(\rho_{a}^{n-1}-\rho_{a}^{n}\right) .
\end{gathered}
$$




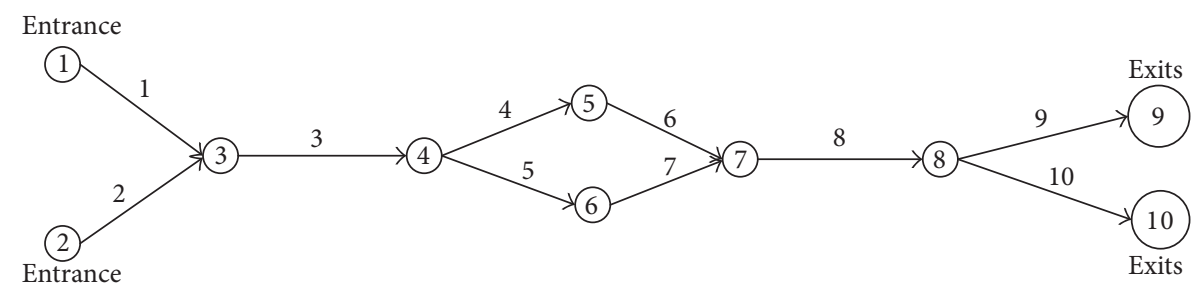

FIGURE 3: Pedestrian network description.

TABLE 1: Network information.

\begin{tabular}{lccccc}
\hline Link & Length $(\mathrm{m})$ & Width $(\mathrm{m})$ & Free walking speed $v_{a}^{m}(\mathrm{~m} / \mathrm{s})$ & Jam density $\rho_{a}^{m}\left(\mathrm{peds} / \mathrm{m}^{2}\right)$ & Initial density $\left(\mathrm{peds} / \mathrm{m}^{2}\right)$ \\
\hline 1 & 50 & 2.5 & 1.5 & 3.8 & 3.8 \\
2 & 50 & 2.5 & 1.5 & 3.8 & 2 \\
3 & 50 & 5 & 1.5 & 3.8 & 3 \\
4 & 50 & 2.5 & 1.5 & 3.8 & 1 \\
5 & 50 & 2.5 & 1.5 & 3.8 & 0.5 \\
6 & 50 & 2.5 & 1.5 & 3.8 & 0.1 \\
7 & 50 & 2.5 & 1.5 & 3.8 & 1.2 \\
8 & 50 & 5 & 1.5 & 3.8 & 0.5 \\
9 & 50 & 2 & 1.5 & 3.8 & 1.1 \\
10 & 50 & 2 & 1.5 & & \\
\hline
\end{tabular}

At present, in (30), all variables except the walking speed $v_{a}^{i}$ are known. The variables $q_{a}^{\text {in }}$ and $q_{a}^{\text {out }}$ can be produced by the NFCM and the section densities $\rho_{a}^{i}$ can be observed and analyzed by the sensors. The walking speed $v_{a}^{i}, i=$ $1,2, \ldots, n-1$, should be adjusted to achieve the uniform link density. There are $n-1$ unknown variables to be determined by solving $n-1$ linear equations, and thus (30) can be easily solved to realize the link flow control objective.

From the physical viewpoint, the network controlled by the NFCM comprises the links which are controlled by LFCM. From the model viewpoint, the LFCM needs the link inflow values produced by the NFCM and the premise of using NFCM is ensured by the LFCM. The feedback control theory is used by the NFCM and LFCM. Therefore, the NFCM and LFCM can be regarded as a hierarchical feedback control model (HFCM) of network-wide pedestrian flow.

\section{Numerical Example: Pedestrian Flow Control in a Hall}

4.1. Network Description and Model Development. To investigate the efficiency of the proposed pedestrian flow control model, we consider a real college university hall in Beijing as a test platform of our control model. The hall is used as a place for important events such as visiting and learning the railway devices and students meetings. The network description of the hall is depicted in Figure 3. The network consists of 10 nodes, 10 links, 2 entrances, and 2 exits. Two divergence and two merge topologies exist in the hall, which makes the selected network general. The network information including the lengths and widths of corridors is listed in Table 1.
For the pedestrian fundamental diagram of links, the Greenshield model [45], which is recommended in the Society of Fire Protection Engineers (SFPE) handbook [46] and [47], is adopted in this paper and can be described as follows:

$$
v_{a}=v_{a}^{m}\left(1-\frac{\rho_{a}}{\rho_{a}^{m}}\right) .
$$

The data including free walking speed, jam density, and initial densities of all links in pedestrian network are also listed in Table 1 . The LOS evaluation standard comes from [16], which is designed to evaluate the LOS of walking facilities in transportation terminals. The LOS standard and the fundamental diagram of pedestrian flow are depicted in Figure 4.

Assume that a large crowd is visiting and has to pass through the hall. There are $4 \mathrm{OD}$ pairs in the network. The corresponding OD demand of the pedestrian network is listed in Table 2. There are two routes to be chosen for each $\mathrm{OD}$, and the route choice probability is equal to 0.5 .

The purpose of this case study is to evaluate the LOS of links during traffic rush hours and the performance of the proposed pedestrian flow control model. Under uncontrolled scenario, the pedestrian flow model in the hall is developed based on the description in Section 2 and then the LOS evaluation results can be obtained and will determine the control decision. If the control is required to improve the LOS of pedestrian network, the pedestrian flow control model is developed based on the description in Section 3 and utilized to provide the control plan for network managers. All computational experiments were conducted in a PC with CPU $2.93 \mathrm{GHz}$, under Windows XP operating system. 

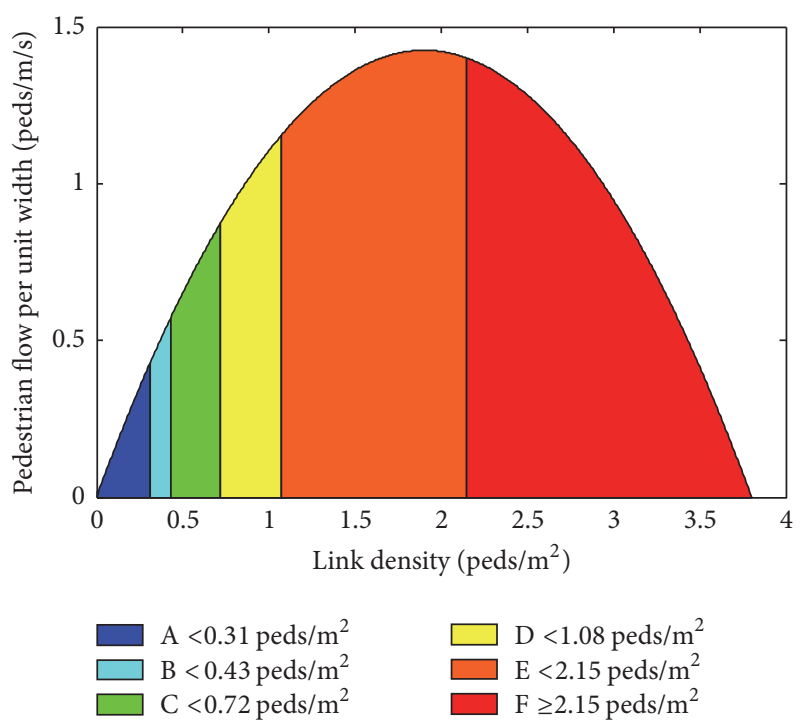

Figure 4: Pedestrian flow fundamental diagram and LOS.

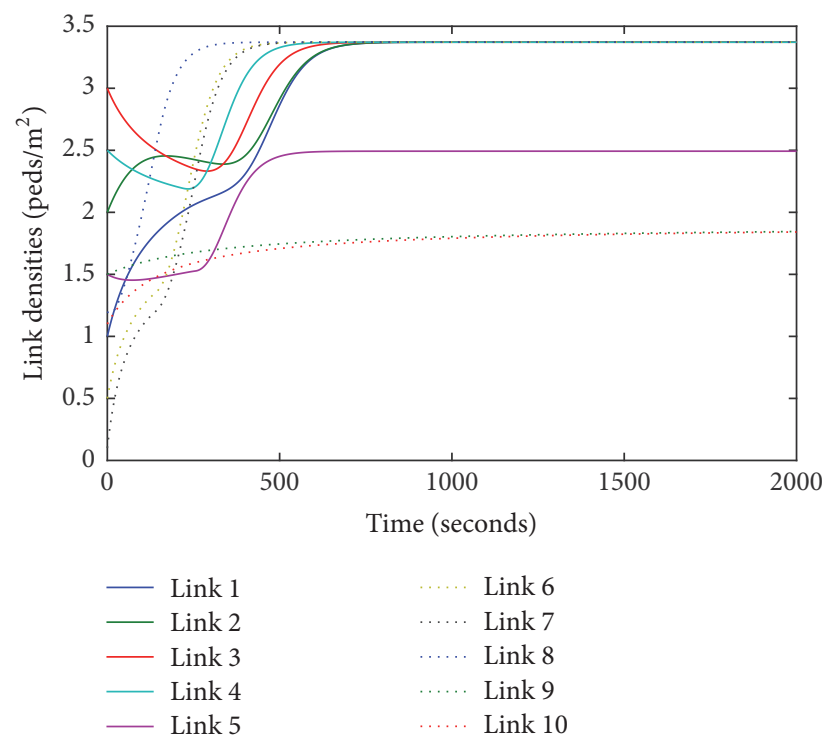

FIGURE 5: Link densities in uncontrolled flow case.

4.2. Model Results and Discussion. Under the self-defined demand scenario, the pedestrian flow in the example network was simulated and the link densities at different time are depicted in Figure 5.

The final link densities can be obtained from the simulation in uncontrolled flow case and the corresponding LOS is evaluated based on the LOS standard as indicated by Figure 4 and the results have been listed in Table 3. The LOS of links 1 to 8 is $\mathrm{F}$ while the LOS of links 8 and 9 is $\mathrm{E}$, as shown in Table 3. The results show that the network traffic performs so seriously that the pedestrian flow control is required to improve the LOS of the network.

The control objective of LOS is assumed to be E and D, respectively, and the corresponding objective densities are 1.9 peds $/ \mathrm{m}^{2}$ and 1.08 peds $/ \mathrm{m}^{2}$ based on (19). The pedestrian
TABLE 2: OD demand of the pedestrian network.

\begin{tabular}{lcc}
\hline Node & 9 & 10 \\
\hline 1 & 3 peds/s & 2 peds $/ \mathrm{s}$ \\
2 & 2 peds/s & 3 peds $/ \mathrm{s}$ \\
\hline
\end{tabular}

TABLE 3: LOS of links in uncontrolled flow case.

\begin{tabular}{lcc}
\hline Link & Final densities $\left(\right.$ peds $\left./ \mathrm{m}^{2}\right)$ & LOS \\
\hline 1 & 3.37 & $\mathrm{~F}$ \\
2 & 3.37 & $\mathrm{~F}$ \\
3 & 3.37 & $\mathrm{~F}$ \\
4 & 3.37 & $\mathrm{~F}$ \\
5 & 2.49 & $\mathrm{~F}$ \\
6 & 3.37 & $\mathrm{~F}$ \\
7 & 3.37 & $\mathrm{~F}$ \\
8 & 3.37 & $\mathrm{~F}$ \\
9 & 1.85 & $\mathrm{E}$ \\
10 & 1.84 & $\mathrm{E}$ \\
\hline
\end{tabular}

flow control model is established based on the description in Section 3. The control gains are chosen from a wide range of gain values. If the value of the control gains $k_{a}$ is too low, then the control objective cannot be realized in a short time. However, if the value of $k_{a}$ is too high, the gain scaling procedure should be done to search the proper gain value repeatedly to ensure the feasibility of (25) and (26), which will lead to additional computing cost and may not realize the real-time control. We have run simulations for a wide range of gain values and found the control gains $k_{a}=0.0065, a=$ $1,2,3, \ldots, 10$, for LOS E and $k_{a}=0.0028, a=1,2,3, \ldots, 10$, for LOS D to be a good tradeoff between high and low gains. The computing time of the pedestrian flow control model ranges from $5.06 \mathrm{~s}$ to $5.28 \mathrm{~s}$, which indicates that the proposed control algorithm can produce the control plan of pedestrian flow in a very short time.

As the result of the network flow control, the link densities converged to the objective densities 1.9 peds $/ \mathrm{m}^{2}$ and 1.08 peds $/ \mathrm{m}^{2}$, which makes the network performance meet the LOS E and LOS D, as indicated by Figure 6. The LOS Eoriented control spends less time than the LOS D-oriented control making the link densities converge to the objective density because larger control gains can result in quicker convergence based on (21) and the control gains of LOS Doriented control $k_{a}=0.0028$ are much lower than LOS E $k_{a}=0.0065$. Based on the control gain selection results of LOS E and LOS D, it can be seen that the LOS D-oriented control needs much more control works than LOS E because the LOS D requires lower density level than LOS E.

The link inflows controlled by NFCM are depicted in Figure 7. In order to realize the objective LOS E, the inflows of links 3 and 8 converged to the steady value of 2.8598 peds/s while the inflows of the other links converged to the steady value of 1.4327 peds/s, as indicated by Figure 7(a). In order to realize the objective LOS D, the inflows of links 3 and 8 converged to the steady value of 2.3192 peds/s while the 


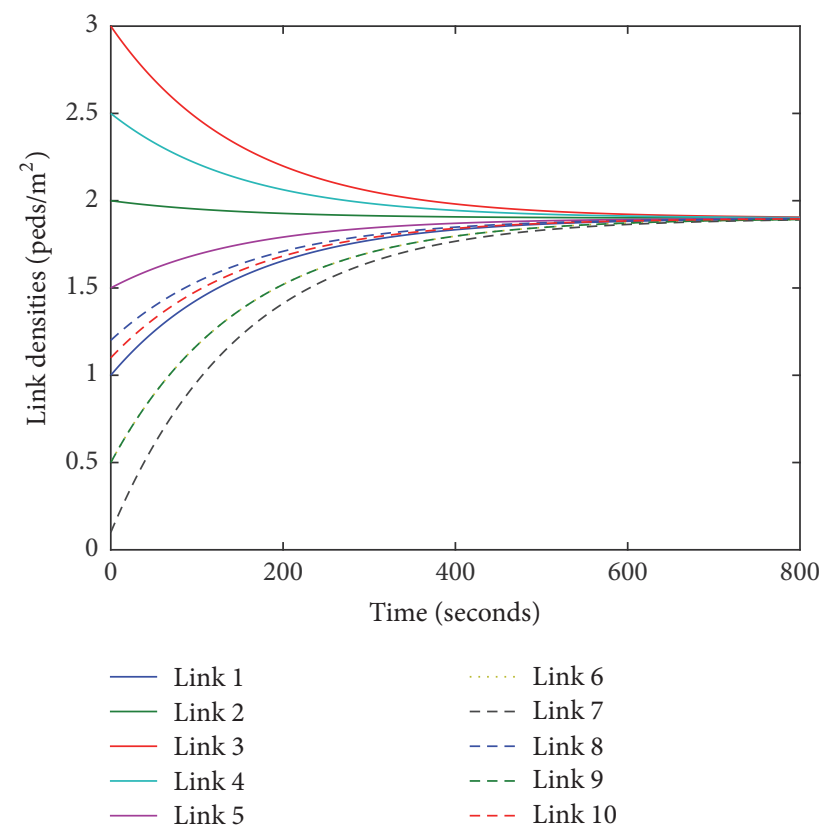

(a) Link densities controlled for LOS E

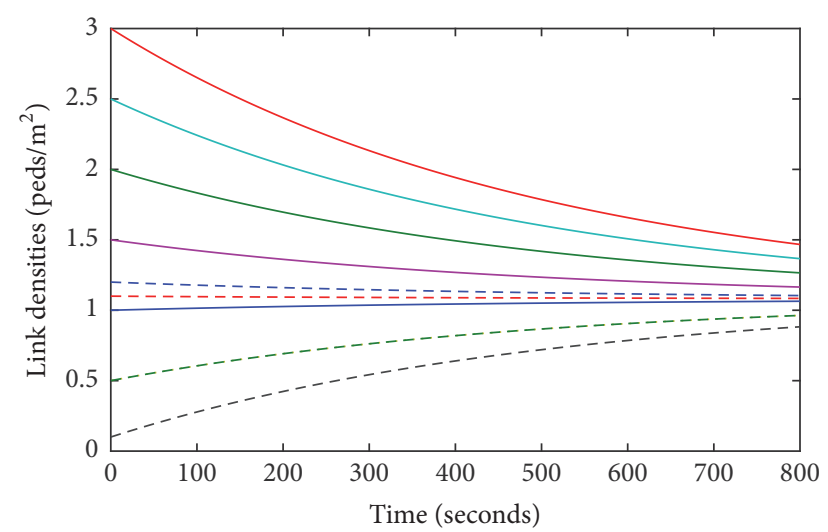

(b) Link densities controlled for LOS D

FIGURE 6: Link densities controlled for LOS E and LOS D.

inflows of the other links converged to the steady value of $1.1596 \mathrm{peds} / \mathrm{s}$, as indicated by Figure 7(b).

In order to prove the necessity of LFCM in the networkwide pedestrian flow control, link 1 is selected and divided into 5 sections, as shown in Figure 8. In the NFCM, the pedestrians are assumed to be homogeneously distributed and the link density is uniform. However, the real fact is that the section densities of link are not the same all the time if only the NFCM is established.

With regard to the LOS E-oriented NFCM, the section densities of link 1 controlled only by NFCM are depicted in Figure 9(a), and the section densities are not equal all the time with only NFCM. For example, the final density values of sections s1, s2, s3, s4, and $\mathrm{s} 5$ are 0.4310 peds $/ \mathrm{m}^{2}, 0.4312 \mathrm{peds} / \mathrm{m}^{2}$, 1.8736 peds $/ \mathrm{m}^{2}, 3.3703$ peds $/ \mathrm{m}^{2}$, and 3.3704 peds $/ \mathrm{m}^{2}$, respectively. Although the average density of 5 sections is equal to the objective density 1.9 peds $/ \mathrm{m}^{2}$, the pedestrians are not to

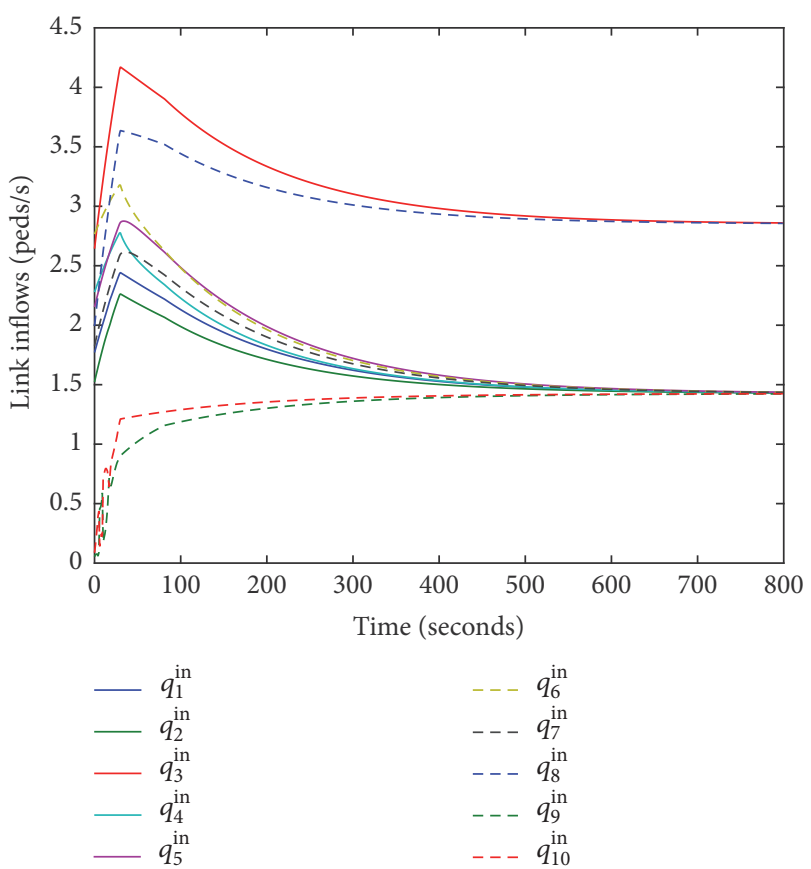

(a) Link inflows controlled for LOS E

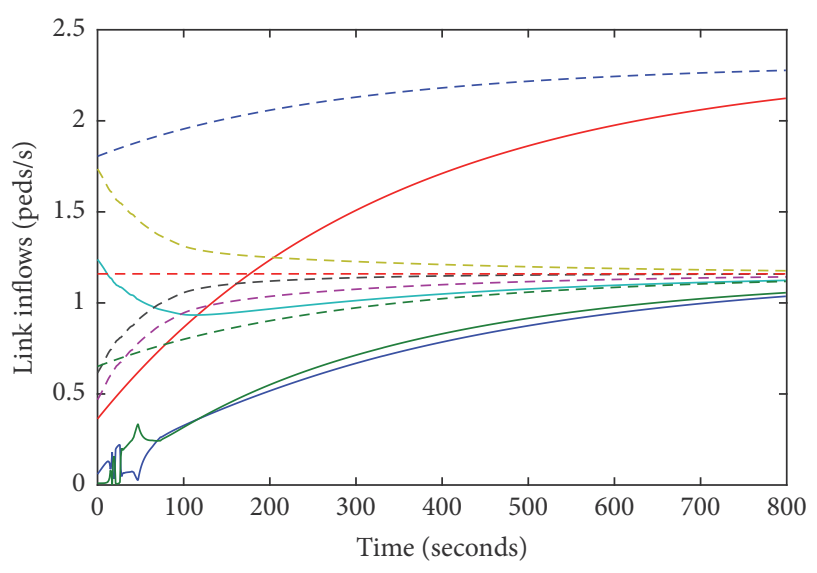

(b) Link inflows controlled for LOS D

FIGURE 7: Link inflows controlled for LOS E and LOS D.

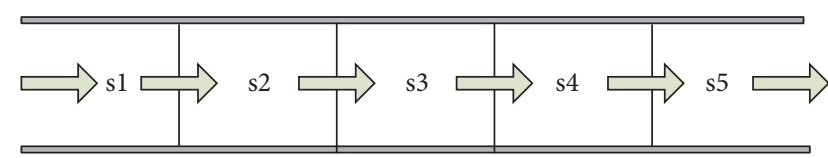

FIGURE 8: 5 sections of link 1.

be homogeneously distributed in link 1 , which is inconsistent with the assumption of NFCM.

With regard to the LOS D-oriented NFCM, a similar phenomenon can be seen from Figure 10(a); the section density values of link 1 are not equal all the time with only NFCM. For example, the final density values of sections s1, s2, s3, $\mathrm{s} 4$, and $\mathrm{s} 5$ are $0.299 \mathrm{peds} / \mathrm{m}^{2}, 0.298 \mathrm{peds} / \mathrm{m}^{2}, 0.297 \mathrm{peds} / \mathrm{m}^{2}$, 0.961 peds $/ \mathrm{m}^{2}$, and 3.502 peds $/ \mathrm{m}^{2}$, respectively. Although the average density of 5 sections is equal to the objective density 


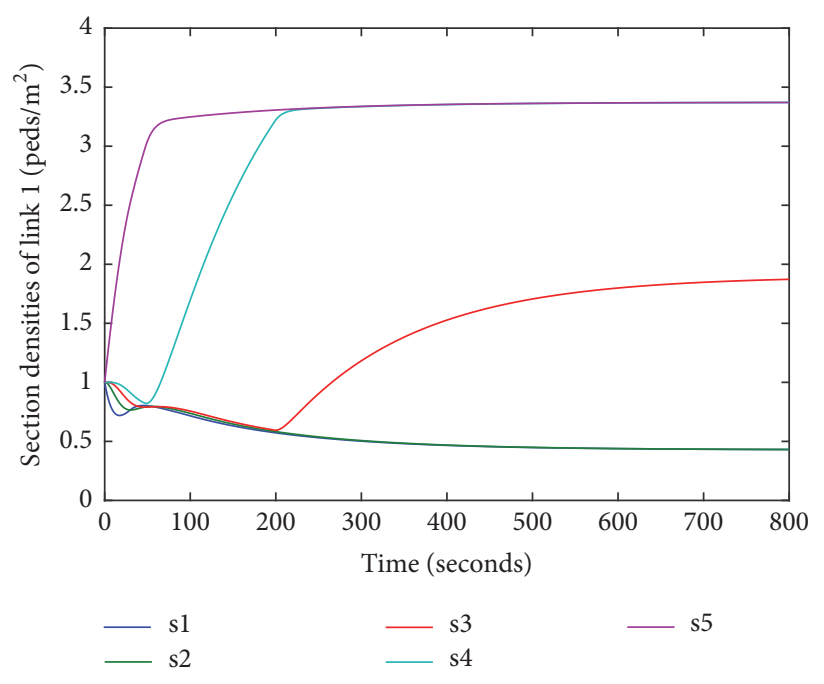

(a) Section densities controlled by NFCM

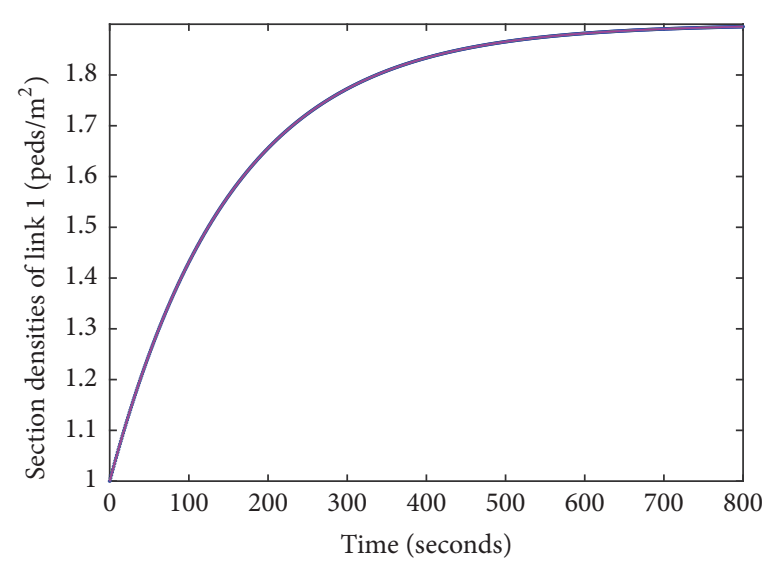

(b) Section densities controlled by NFCM and LFCM

Figure 9: Section densities controlled for LOS E.

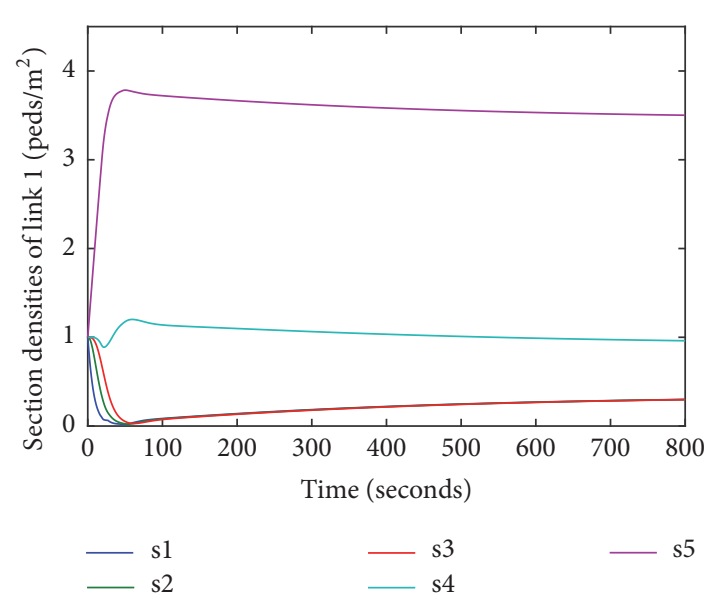

(a) Section densities controlled by NFCM

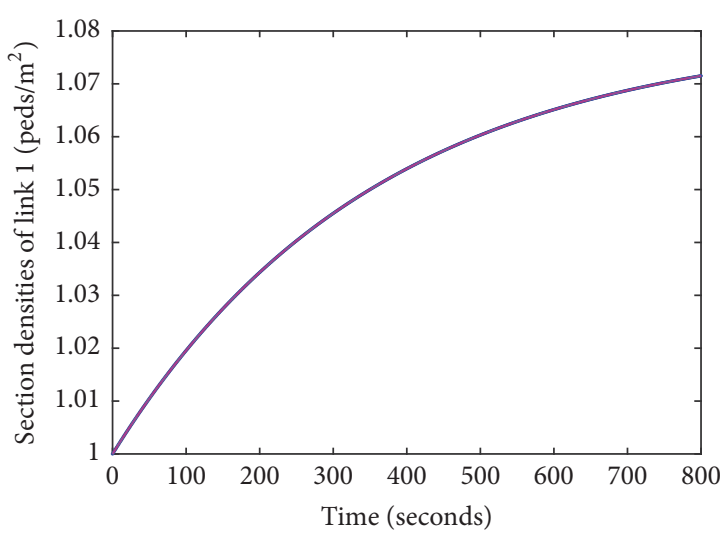

(b) Section densities controlled by NFCM and LFCM

FIgURE 10: Section densities controlled for LOS D.

1.08 peds $/ \mathrm{m}^{2}$, the pedestrians are not to be homogeneously distributed in link 1, which is also inconsistent with the assumption of NFCM. Therefore, the proposed LFCM is required to ensure the NFCM's feasibility.

In the LOS E-oriented LFCM, the control gains $K_{1}^{i}=$ 0.001 for $i=1,2, \ldots, 5$. Figure 9 (b) shows the section densities of link 1 at different time after the control plan produced by LFCM is executed. The curves of section density of link 1 overlapped in Figure 9(b), which indicates that the time-dependent values of section densities are equal and the uniform link density of NFCM is ensured by the LFCM. The controlled walking speeds of pedestrians in link $1, v_{1}^{1}, v_{1}^{2}, v_{1}^{3}$, and $v_{1}^{4}$, are depicted in Figure 11(a). The walking speed has converged to the steady value of $0.6 \mathrm{~m} / \mathrm{s}$, which describes the speed control strategy for LOS E-oriented link flow control in detail.
In the LOS D-oriented LFCM, the control gains $K_{1}^{i}=$ 0.001 for $i=1,2, \ldots, 5$. Figure 10(b) shows the section densities of link 1 at different time after the LFCM is executed. The curves of section density of link 1 overlapped in Figure 10(b), which indicates that the time-dependent section densities are equal and the uniform link density is realized by the LFCM. The controlled walking speeds in link $1, v_{1}^{1}, v_{1}^{2}, v_{1}^{3}$, and $v_{1}^{4}$, are also depicted in Figure 11(b). The walking speeds are also converging to the steady value of $0.6 \mathrm{~m} / \mathrm{s}$, which describes the speed control strategy for LOS D-oriented link flow control in detail. Therefore, the LFCM can realize the uniform link density and thus ensure the NFCM's feasibility; at the same time, the LFCM can realize the full and even use of link capacity.

All in all, the hierarchical feedback control model (HFCM), which integrates NFCM and the LFCM, can make 


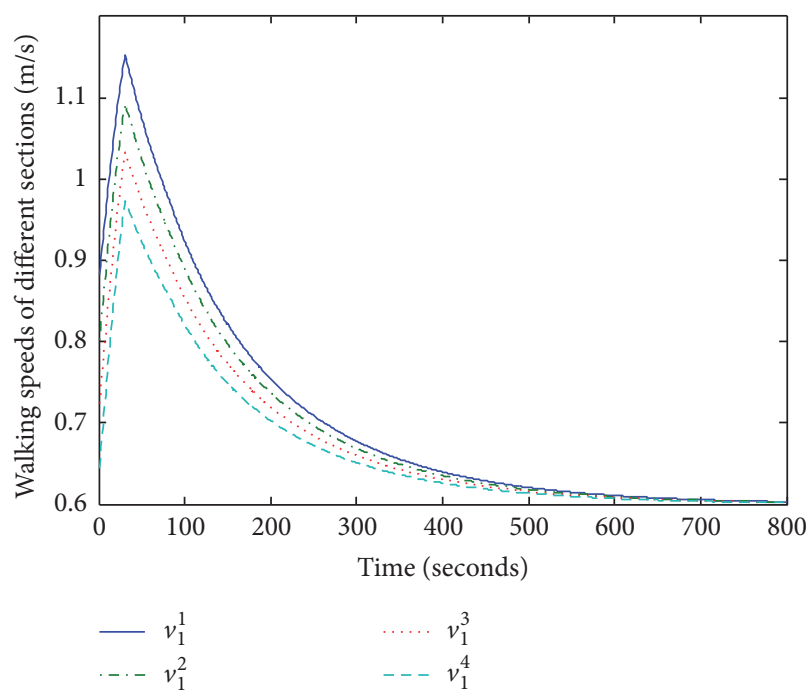

(a) Walking speeds controlled for LOS E

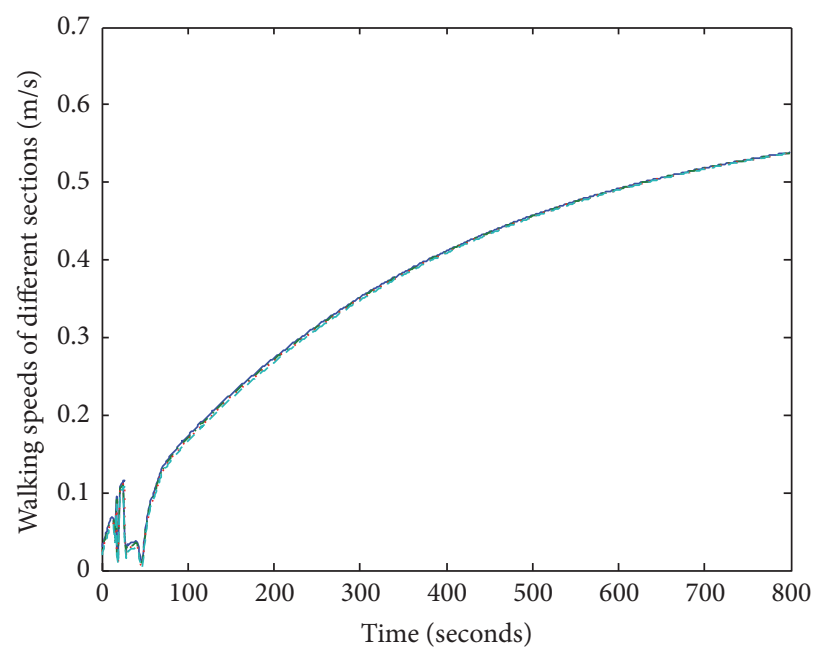

(b) Walking speeds controlled for LOS D

FIGURE 11: Walking speeds controlled by LFCM.

the pedestrian network meet the objective LOS requirement by controlling the link inflows and adjusting the walking speed of pedestrians.

\section{Conclusion and Extension}

In order to realize the real-time control of pedestrian flow in network and provide crucial support for the LOS-based crowd management, this paper presented a hierarchical feedback control model (HFCM) of network-wide pedestrian flow.

First, this paper presents a cell transmission based network-wide pedestrian flow model by taking the links as the minimum analytical units. It realizes a holistic assessment of the network performance in order to support the decisionmaking about pedestrian flow control, as it provides us with a big-picture averaged view of the network-wide pedestrian flow state. Based on the conservation of mass, the collection of ODEs is used to represent the state-space equations of network-wide pedestrian flow and that makes the establishment of pedestrian flow control model easier than the PDEbased model. Additionally, the proposed pedestrian flow model can be easily adapted to a wide spectrum of public facility configurations, as the flow model for different topologies has been developed in this paper and the numerical example also proved its flexibility in achieving pedestrian flow simulation in general network.

Secondly, the network flow control model (NFCM) is firstly proposed to find the optimal link inflows to ensure the number of pedestrians in the link satisfies the LOS requirement. In order to realize the uniform link density and make full and even use of the link capacity, the link flow control model (LFCM) has been proposed to adjust the walking speed of pedestrians by dividing the link into many sections. The LFCM uses the link inflows from the NFCM and the premise of using NFCM is ensured by the LFCM in turn. The two models control the pedestrian flow in a cooperative way and are integrated as the HFCM of networkwide pedestrian flow.

Finally, the proposed HFCM is applied to control the pedestrian flow in a hall and the model results show that the HFCM can find the optimal control plan to trade off the benefits of pedestrians and managers. The higher LOS requirement needs much more control works, which is also consistent with the reality. The HFCM can be regarded as a general control model for network-wide crowd management. The computing time of the proposed model is about 5 seconds which indicates that the control plan can be generated in real time.

The proposed control algorithm utilizes the pedestrian density data to compute the optimal inflows and walking speeds and thus it depends on the real-time capture of link densities, which can be realized by the videos or sensors. Therefore, the proposed control model can be integrated with the intelligent devices such as videos, sensors [48], and wireless communication network to realize the LOS-based intelligent control of network-wide pedestrian flow.

Future research includes the comparison of the results obtained by the proposed pedestrian flow control model against both actual data and the outcomes of other software packages that have been used to simulate the pedestrian flow under control, for example, Anylogic and Legion. Moreover, there is opportunity for model enhancements towards

(1) the extension of the proposed control model to address the control of multidirectional pedestrian flow in a more general network with much more kinds of facilities such as stairs, walkways, escalators, and slopes;

(2) the extension of the proposed model to address the pedestrian flow control with accounting for the presence of uncertainties, such as the uncertain link 
capacity and traffic demand, the error between the inflow control plan and the actual controlled link inflow, and the pedestrians' willingness to obey the control command;

(3) the extension of the proposed model to address the flow control with accounting for the dynamic route choice behavior of pedestrians in the control process;

(4) the extension of the proposed control model to address the regulation of traffic flow in freeway networks which is controlled by the traffic signal lights and speed limiting.

\section{Competing Interests}

The authors declare no competing interests.

\section{Authors' Contributions}

Zhe Zhang analyzed the previous studies about pedestrian flow control and built the pedestrian flow model and the control model. Limin Jia gave suggestions and helped complete and wrote the manuscript.

\section{Acknowledgments}

The research was supported by the Fundamental Research Funds for the Central Universities (no. 2016YJS075) and the National Natural Science Foundation of China (no. 71171015).

\section{References}

[1] E. Ben-Elia and D. Ettema, "Rewarding rush-hour avoidance: a study of commuters' travel behavior," Transportation Research Part A: Policy and Practice, vol. 45, no. 7, pp. 567-582, 2011.

[2] X.-Y. Xu, J. Liu, H.-Y. Li, and J.-Q. Hu, "Analysis of subway station capacity with the use of queueing theory," Transportation Research Part C: Emerging Technologies, vol. 38, pp. 28-43, 2014.

[3] J. van den Heuvel, A. Voskamp, W. Daamen, and S. P. Hoogendoorn, "Using bluetooth to estimate the impact of congestion on pedestrian route choice at train stations," in Traffic and Granular Flow '13, pp. 73-82, Springer International, 2015.

[4] C. Shi, M. Zhong, X. Nong, L. He, J. Shi, and G. Feng, "Modeling and safety strategy of passenger evacuation in a metro station in China," Safety Science, vol. 50, no. 5, pp. 1319-1332, 2012.

[5] J. Y. S. Lee, P. K. Goh, and W. H. K. Lam, "New level-ofservice standard for signalized crosswalks with bi-directional pedestrian flows," Journal of Transportation Engineering, vol. 131, no. 12, pp. 957-960, 2005.

[6] K. M. Kim, S.-P. Hong, S.-J. Ko, and D. Kim, "Does crowding affect the path choice of metro passengers?" Transportation Research Part A: Policy and Practice, vol. 77, pp. 292-304, 2015.

[7] F. Delgado, J. C. Muñoz, R. Giesen, and A. Cipriano, "Real-time control of buses in a transit corridor based on vehicle holding and boarding limits," Transportation Research Record: Journal of the Transportation Research Board, vol. 2090, pp. 59-67, 2009.

[8] F. Delgado, J. C. Munoz, and R. Giesen, "How much can holding and/or limiting boarding improve transit performance?" Transportation Research Part B: Methodological, vol. 46, no. 9, pp. 1202-1217, 2012.
[9] X.-Y. Xu, J. Liu, H.-Y. Li, and M. Jiang, “Capacity-oriented passenger flow control under uncertain demand: algorithm development and real-world case study," Transportation Research Part E: Logistics and Transportation Review, vol. 87, pp. 130-148, 2016.

[10] S. Seriani and R. Fernandez, "Pedestrian traffic management of boarding and alighting in metro stations," Transportation Research Part C: Emerging Technologies, vol. 53, pp. 76-92, 2015.

[11] C. Norris, M. McCahill, and D. Wood, "The growth of CCTV: a global perspective on the international diffusion of video surveillance in publicly accessible space," Surveillance \& Society, vol. 2, no. 2-3, 2002.

[12] C. Kim, D. K. Kim, S. Y. Kho, S. Kang, and K. Chung, "Dynamically determining the toll plaza capacity by monitoring approaching traffic conditions in real-time," Applied Sciences, vol. 6 , no. 3, p. 87, 2016.

[13] F. Kaakai, S. Hayat, and A. El Moudni, "A hybrid Petri netsbased simulation model for evaluating the design of railway transit stations," Simulation Modelling Practice and Theory, vol. 15, no. 8, pp. 935-969, 2007.

[14] I. E. Manataki and K. G. Zografos, "A generic system dynamics based tool for airport terminal performance analysis," Transportation Research Part C: Emerging Technologies, vol. 17, no. 4, pp. 428-443, 2009.

[15] L. Hu, Y. Jiang, J. Zhu, and Y. Chen, "A PH/PH(n)/C/C statedependent queuing model for metro station corridor width design," European Journal of Operational Research, vol. 240, no. 1, pp. 109-126, 2015.

[16] A. R. Danaher, Transit Capacity and Quality of Service Manual, Transportation Research Circular, 2003.

[17] G. Hoy, E. Morrow, and A. Shalaby, "Use of agent-based crowd simulation to investigate the performance of large-scale intermodal facilities," Transportation Research Record: Journal of the Transportation Research Board, vol. 2540, pp. 20-29, 2016.

[18] D. Helbing, I. Farkas, and T. Vicsek, "Simulating dynamical features of escape panic," Nature, vol. 407, no. 6803, pp. 487$490,2000$.

[19] K. Takimoto, Y. Tajima, and T. Nagatani, "Effect of partition line on jamming transition in pedestrian counter flow," Physica A: Statistical Mechanics and Its Applications, vol. 308, no. 1-4, pp. 460-470, 2002.

[20] F. R. B. Cruz, J. M. Smith, and D. C. Queiroz, "Service and capacity allocation in $\mathrm{M} / \mathrm{G} / \mathrm{c} / \mathrm{c}$ state-dependent queueing networks," Computers \& Operations Research, vol. 32, no. 6, pp. 1545-1563, 2005.

[21] M. E. O'Kelly, "Routing traffic at hub facilities," Networks and Spatial Economics, vol. 10, no. 2, pp. 173-191, 2010.

[22] L. Feng and E. Miller-Hooks, "A network optimization-based approach for crowd management in large public gatherings," Transportation Research Part C: Emerging Technologies, vol. 42, pp. 182-199, 2014.

[23] R. Khalid, M. A. Baten, M. K. M. Nawawi, and N. Ishak, "Analyzing and optimizing pedestrian flow through a topological network based on M/G/C/C and network flow approaches," Journal of Advanced Transportation, vol. 50, no. 1, pp. 96-119, 2016.

[24] P. Kachroo, S. J. Al-Nasur, S. A. Wadoo, and A. Shende, Pedestrian Dynamics: Feedback Control of Crowd Evacuation, Springer Science \& Business Media, 2008.

[25] S. A. Wadoo and P. Kachroo, "Feedback control of crowd evacuation in one dimension," IEEE Transactions on Intelligent Transportation Systems, vol. 11, no. 1, pp. 182-193, 2010. 
[26] A. Shende, M. P. Singh, and P. Kachroo, "Optimization-based feedback control for pedestrian evacuation from an exit corridor," IEEE Transactions on Intelligent Transportation Systems, vol. 12, no. 4, pp. 1167-1176, 2011.

[27] A. Shende, M. P. Singh, and P. Kachroo, "Optimal feedback flow rates for pedestrian evacuation in a network of corridors," IEEE Transactions on Intelligent Transportation Systems, vol. 14, no. 3, pp. 1053-1066, 2013.

[28] G.-X. Zeng and Y. Xue, "Application of the quasi-sliding-mode control to traffic bottleneck in pedestrian channel," Acta Physica Sinica, vol. 1, article 58, 2011.

[29] S. A. Wadoo, "Sliding mode control of crowd dynamics," IEEE Transactions on Control Systems Technology, vol. 21, no. 3, pp. 1008-1015, 2013.

[30] M. J. Lighthill and G. B. Whitham, "On kinematic waves. II. A theory of traffic flow on long crowded roads," Proceedings of the Royal Society. London. A. Mathematical, Physical and Engineering Sciences, vol. 229, pp. 317-345, 1955.

[31] C. F. Daganzo, "The cell transmission model, part II: network traffic," Transportation Research Part B: Methodological, vol. 29, no. 2, pp. 79-93, 1995.

[32] L. Muñoz, X. Sun, R. Horowitz, and L. Alvarez, "Traffic density estimation with the cell transmission model," in Proceedings of the IEEE American Control Conference, vol. 5, pp. 3750-3755, 2003.

[33] A. Sumalee, R. X. Zhong, T. L. Pan, and W. Y. Szeto, "Stochastic cell transmission model (SCTM): a stochastic dynamic traffic model for traffic state surveillance and assignment," Transportation Research Part B: Methodological, vol. 45, no. 3, pp. 507-533, 2011.

[34] F. S. Hänseler, M. Bierlaire, B. Farooq, and T. Mühlematter, "A macroscopic loading model for time-varying pedestrian flows in public walking areas," Transportation Research Part B: Methodological, vol. 69, pp. 60-80, 2014.

[35] M. Asano, A. Sumalee, M. Kuwahara, and S. Tanaka, "Dynamic cell transmission-based pedestrian model with multidirectional flows and strategic route choices," Transportation Research Record, no. 2039, pp. 42-49, 2007.

[36] X. Zhang and G.-L. Chang, "Optimal control strategies with an extended cell transmission model for massive vehicularpedestrian mixed flows in the evacuation zone," Journal of Advanced Transportation, vol. 48, no. 8, pp. 1030-1050, 2014.

[37] F. R. B. Cruz, J. MacGregor Smith, and R. O. Medeiros, "An $M / G / C / C$ state-dependent network simulation model," Computers \& Operations Research, vol. 32, no. 4, pp. 919-941, 2005.

[38] C. M. J. Tampère, R. Corthout, D. Cattrysse, and L. H. Immers, "A generic class of first order node models for dynamic macroscopic simulation of traffic flows," Transportation Research Part B: Methodological, vol. 45, no. 1, pp. 289-309, 2011.

[39] W. L. Jin and H. M. Zhang, "On the distribution schemes for determining flows through a merge," Transportation Research Part B: Methodological, vol. 37, no. 6, pp. 521-540, 2003.

[40] D. Ni and J. D. Leonard II, "A simplified kinematic wave model at a merge bottleneck," Applied Mathematical Modelling, vol. 29, no. 11, pp. 1054-1072, 2005.

[41] M. Campanella, R. Halliday, S. Hoogendoorn, and W. Daamen, "Managing large flows in metro stations: the new year celebration in copacabana," IEEE Intelligent Transportation Systems Magazine, vol. 7, no. 1, pp. 103-113, 2015.

[42] J. Fruin, Designing for Pedestrians, Public Transportation United States, 1992.
[43] S. P. Hoogendoorn and P. H. L. Bovy, "Pedestrian route-choice and activity scheduling theory and models," Transportation Research Part B: Methodological, vol. 38, no. 2, pp. 169-190, 2004.

[44] Z. Liu, S. Wang, W. Chen, and Y. Zheng, "Willingness to board: a novel concept for modeling queuing up passengers," Transportation Research Part B: Methodological, vol. 90, pp. 7082, 2016.

[45] B. D. Greenshields, W. S. Channing, H. H. Miller et al., "A study of traffic capacity," in Proceedings of the 14th Annual Meeting of the Highway Research Board, Washington, DC, USA, 1935.

[46] M. J. Hurley, D. T. Gottuk, J. R. Hall et al., "SFPE handbook of fire protection engineering," in Industrial Safety and Environmental Protection, vol. 29, pp. 487-500, 2016.

[47] S. Al-nasur and P. Kachroo, "A microscopic-to-macroscopic crowd dynamic model," in Proceedings of the IEEE Intelligent Transportation Systems Conference (ITSC '06), pp. 606-611, Toronto, Canada, September 2006.

[48] T.-J. Ho and M.-J. Chung, "Information-aided smart schemes for vehicle flow detection enhancements of traffic microwave radar detectors," Applied Sciences, vol. 6, no. 7, article 196, 2016. 


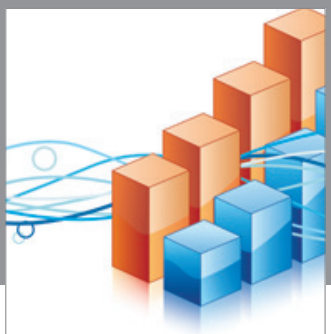

Advances in

Operations Research

vatem alat4

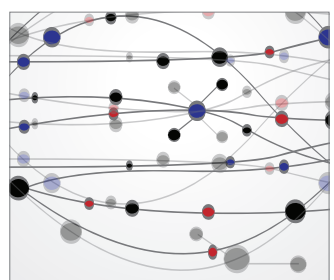

\section{The Scientific} World Journal
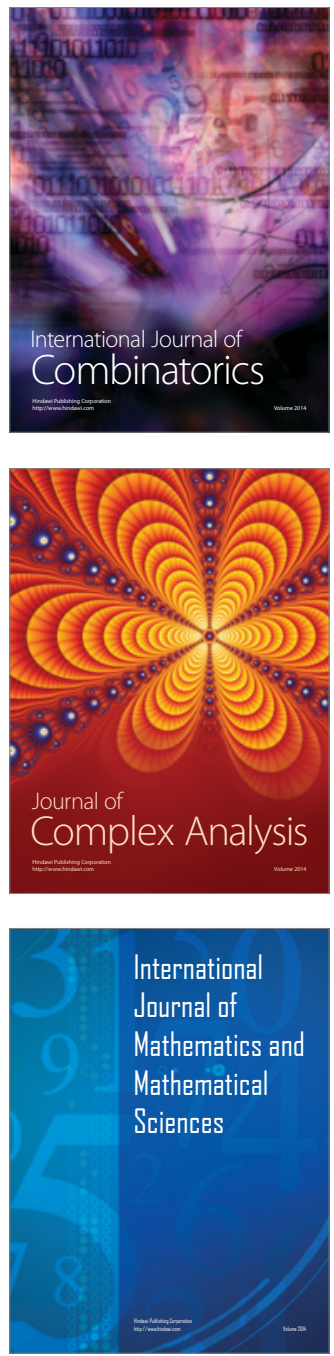
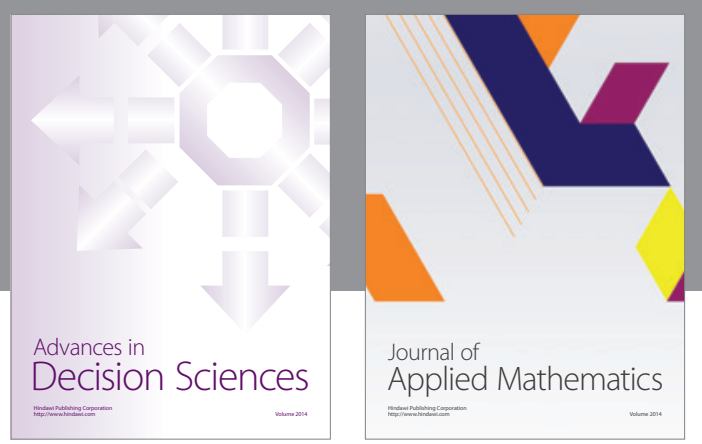

Algebra

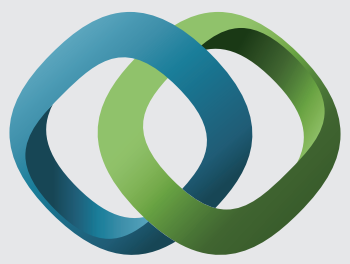

\section{Hindawi}

Submit your manuscripts at

http://www.hindawi.com
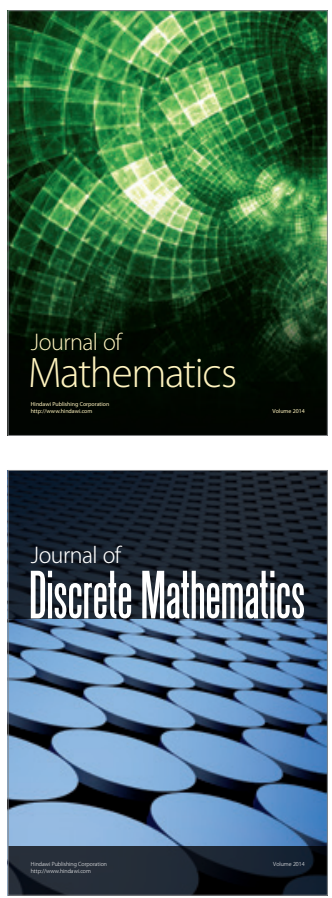

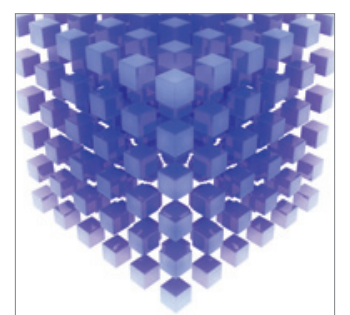

Mathematical Problems in Engineering
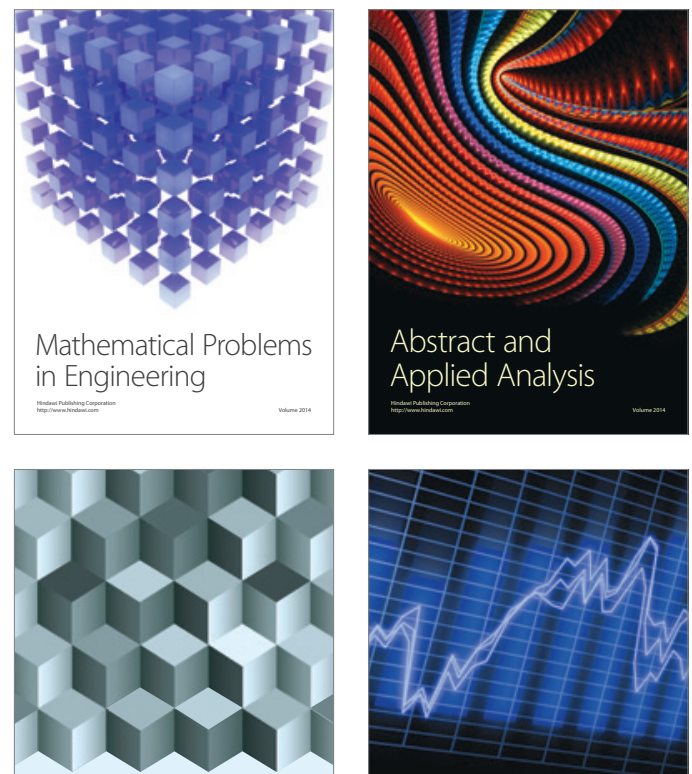

Journal of

Function Spaces

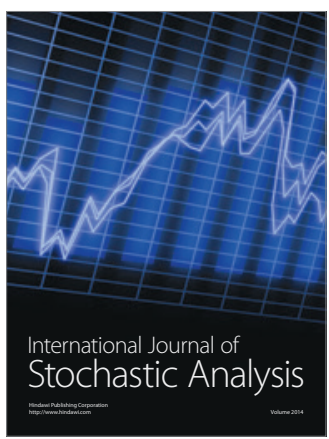

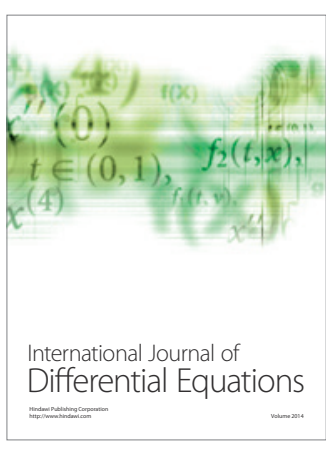
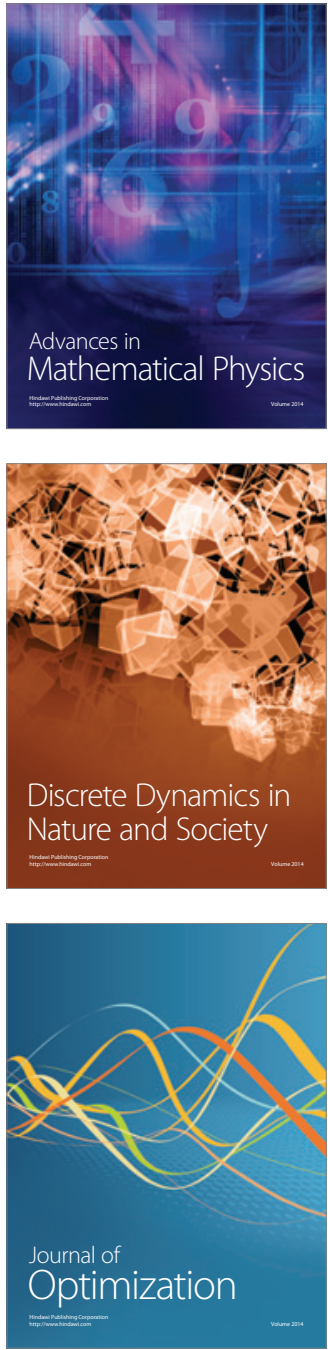